\title{
SCHEDULING ÓPTIMO DE PROCESOS BATCH DE DURACIÓN INTERDEPENDIENTE
}

\author{
Carlos G. Palacín*,a , Pablo Riquelme ${ }^{\mathrm{a}, \mathrm{b}}$, Cesar de Prada ${ }^{\mathrm{a}, \mathrm{b}}$ \\ ${ }^{a}$ Dpto. de Ingeniería de Sistemas y Automática. EII, sede Mergelina. Universidad de Valladolid \\ ${ }^{\mathrm{b}}$ Instituto de Procesos Sostenibles (IPS). C/Real de Burgos, S/N. Universidad de Valladolid \\ C/Real de Burgos S/N, Valladolid, 47011 \\ \{carlos.gomez@autom.uva.es | pablo.riquelme@uva.es|prada@autom.uva.es\}
}

\section{Resumen}

Cuando distintos equipos similares trabajan de manera concurrente compartiendo un recurso, los perfiles de trabajo de cada uno se pueden ver afectados por un consumo simultáneo. De esta manera, surge un problema de programación de las tareas o scheduling, para encontrar la planificación temporal y de asignación óptima que tenga en cuenta estas interdependencias entre los equipos. Este problema se puede complicar aún más si la duración de los procesos depende de los tipos de producto a tratar. En este trabajo se presenta una herramienta que optimiza el scheduling de una sección de esterilizado de una planta de conservas, donde el vapor se comparte entre distintos autoclaves paralelos. Los autoclaves se emplean en el proceso de esterilizado de las latas de conserva, que tienen distintos perfiles de calentamiento dependiendo del producto que contienen y de la forma de las mismas. Con un enfoque de horizonte deslizante, la herramienta puede ser ejecutada en tiempo real y asi poder adaptarse a los cambios que muy probablemente se producirán a lo largo de los turnos de trabajo. El modelo se genera como un problema de programación lineal mixta-entera, y con un planteamiento de slots continuos. Se presenta una mejora de los algoritmos de precedencia que se han venido utilizando en la literatura. Finalmente, se muestran con un ejemplo los resultados obtenidos que demuestran la posibilidad de su implantación real en la planta.

Palabras clave: Autoclaves, RTO, horizonte deslizante, esterilización.

\section{INTRODUCCIÓN}

En la industria alimentaria suelen aparecer procesos por lotes o procesos batch durante alguna fase de la producción continua. Si los equipos requeridos para llevar a cabo dichos procesos discretos se comparten entre distintas líneas de producción, aparece un problema de planificación o scheduling. Estos problemas se complican si los diferentes productos a tratar tienen perfiles de procesamiento distintos, o bien si existe algún recurso necesario para la ejecución del que se tiene suministro limitado 5. En este caso, los planteamientos típicos de la literatura suelen realizar una reserva de la cantidad necesaria de recursos al inicio del proceso, y la liberación de la misma al finalizar [10]. Si bien esta aproximación es útil para los problemas de planificación a largo plazo, peca de agresiva, pues los recursos no se emplean durante todo el proceso de igual manera. Del mismo modo, si al compartir los recursos los procesos alteran de alguna manera su comportamiento, pero pueden ser ejecutados igualmente, la reserva de los recursos estaría eliminando posibles soluciones, incluida la óptima en algún caso, del espacio factible del modelo.

Para enfrentarse a dichos problemas, existen sendas alternativas: bien se pueden reservar los recursos siguiendo un perfil temporal variable, frente a la reserva del máximo necesario durante todo el proceso, lo que permite maximizar el uso de los recursos compartidos ajustando mejor el scheduling de los procesos 9 ; o bien se puede diseñar el modelo teniendo en cuenta que los equipos ven afectado su comportamiento al compartir dichos recursos 8]. Este trabajo aumenta los resultados obtenidos en la segunda vertiente en trabajos previos, donde la alteración entre los equipos se aproximaba como un máximo, mientras que en este caso se calcula como relativa al tiempo de solapamiento de los procesos, lo que supone una aproximación más realista.

Frente a las estrategias clásicas de scheduling que trabajan con largos horizontes de predicción [1, 3, considerando a largos horizontes jornadas completas de trabajo, presentamos una herramienta que tiene un horizonte corto pero se ejecuta de manera iterativa con una alta frecuencia para poder adaptarse a los cambios en las líneas de producción. Para comprobar la validez del enfoque se presenta un ejemplo en una planta de conservas donde se produce un proceso de esterilizado de tipo batch en medio de la fabricación continua de las latas de conserva.

El resto del artículo está organizado de la siguiente manera: la planta industrial concreta se describe en la sección siguiente, 2, en la sección 3 se mues- 
tra el modelo matemático que resuelve el problema; después, se muestran resultados reales con un ejemplo de ejecución; y, por último, en la sección 5 se analizan los resultados obtenidos y se extraen las conclusiones del estudio correspondientes.

\section{CASO DE ESTUDIO}

La planta industrial sobre la que se ha planteado el problema es una planta conservera. A la misma llegan las piezas de pescado o marisco a tratar y, tras ser limpiadas, preparadas e introducidas en los envases de conserva (latas), éstos se terminan de rellenar con distintos conservantes dependiendo del producto final: aceite de oliva, escabeche, salsa de tomate, etcétera. Después, las latas son selladas herméticamente con las tapas y se disponen en grandes carros industriales metálicos de manera automática por las líneas de sellado. Cada línea de procesado sólo trabaja con un tipo de producto cada vez, por lo que los carros sólo tienen un tipo de conserva en ellos; es decir, se puede hablar de tipo de carro, refiriéndose al tipo de conserva que transportan. Los carros se colocan en las línea de sellado de manera indistinta según van siendo necesitados.

Tras el sellado, las latas deben ser sometidas a un proceso de esterilizado. Este proceso se lleva a cabo en unos autoclaves o esterilizadores industriales donde se introducen los carros llenos. Cada esterilizador tiene una capacidad máxima de carros. Con el sellado de las latas se inicia una cuenta atrás hasta que se inicia su proceso de esterilizado. Esta cuenta atrás no puede superar un tiempo máximo para evitar el crecimiento de aminas y microorganismos en el producto, que afectarían a su seguridad sanitaria. Debido a este límite en el tiempo de espera, se pueden llegar a producir procesos de esterilización con un número muy reducido de carros a fin de evitar los gastos que supondría la pérdida de los carros por motivos sanitarios, nótese que en cada carro puede llegar a haber miles de latas dependiendo del formato del envase. El proceso de esterilizado en sí consiste en mantener a una temperatura mínima las latas durante un tiempo mínimo. Esta relación tiempotemperatura, de ahora en adelante receta, viene prefijada por unos estándares de organismos estatales de seguridad alimentaria y es relativo a cada tipo de lata.

Existe una serie de autoclaves totalmente equivalentes en paralelo para dar servicio a todas las líneas de sellado. Cuando un carro es liberado de las líneas de sellado es empujado manualmente hasta el autoclave más cercano que esté libre o en el que ya se hayan introducido previamente carros del mismo tipo. Una vez lleno, o bien si alguno de los carros incluidos va a superar el tiempo máximo de espera, el autoclave se cierra y se inicia el correspondiente proceso de esterilizado. Tras lo cual, el autoclave se debe vaciar alimentando a las líneas siguientes, que corresponden a las líneas de empaquetado, donde las latas se organizan en grupos dependiendo del tipo. Estas líneas de empaquetado, de igual manera que las líneas de sellado, vacían los carros de manera automática. Cuando un carro se vacía por completo, se envía al punto de partida para ser nuevamente llenado en las líneas de sellado.

Los autoclaves están conectados a un anillo de vapor, que es el principal recurso que se emplea para calentar los equipos. El proceso de esterilizado se puede dividir en tres fases (véase figura 1): una fase de calentamiento inicial, que aumenta la temperatura del equipo hasta el valor fijado; una etapa de mantenimiento, que conserva la temperatura constante durante el tiempo necesario marcado por la receta; y una etapa de enfriado final, que reduce la temperatura dentro del equipo para poder vaciarlo de manera más rápida. El consumo de vapor principalmente se realiza durante la primera etapa. Sin embargo, durante la etapa de mantenimiento el consumo de vapor se reduce puesto que el equipo tiene una gran inercia térmica. Por último, en la etapa de enfriamiento el consumo de vapor es nulo, usando agua fría para reducir la temperatura en el interior. Si dos equipos coinciden durante la primera fase la duración de ambas fases se ve alterada, aumentando debido a la reducción del flujo de vapor a cada uno de los equipos y, por consiguiente, el tiempo global de los procesos de esterilizado se ve afectado para ambos lotes. Si alguno de los equipos se encuentra en alguna de las otras dos fases, los tiempos no se ven afectados, o bien la variación es despreciable.

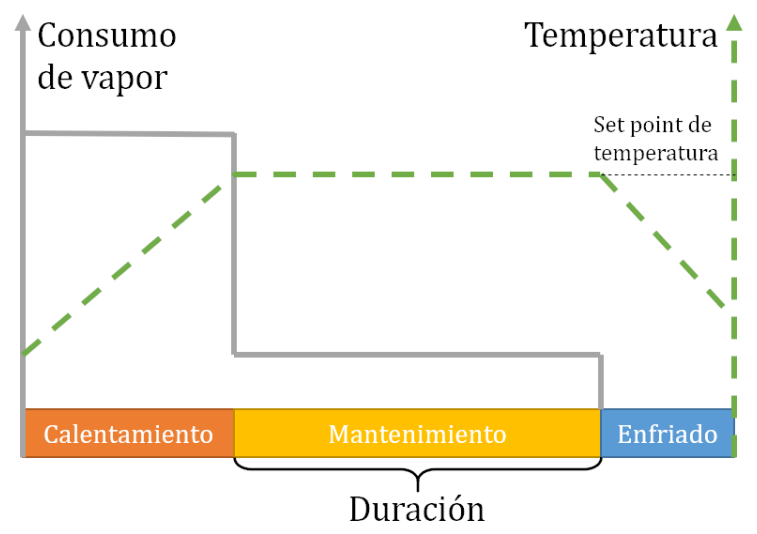

Figura 1: Representación gráfica de las fases del proceso de esterilizado

Debido a restricciones de espacio, no todas las 
líneas de sellado pueden alimentar cualquier autoclave, si no que existe una matriz de posibles conexiones. Actualmente, los autoclaves se llenan sólo de un tipo de carro, debiendo esperar a que se alcance la capacidad del equipo para ponerlo en marcha, o bien a que algún carro esté proximo a su espera máxima. Con el algoritmo desarrollado se permite mezclar carros hasta una diferencia de tiempo prefijada. Esto se deberá evitar, aunque permitirá tratar los carros a tiempo reduciendo las pérdidas que se ocasionarían por someter a pocos carros al tratamiento térmico.

Cuando el proceso de esterilizado termina, los carros son extraídos de manera manual de los autoclaves. Por esta razón, aunque se permite la mezcla de carros de tipos distintos, no se permite que se mezclen demasiadas referencias en el mismo autoclave, a fin de no complicar la tarea de disposición de los carros a las líneas de empaquetado posteriores.

\section{MODELO MATEMÁTICO}

El sistema se ha diseñado con predicados lógicos, si bien se ha realizado una traducción a un modelo matemático para facilitar su codificación en lenguaje máquina. Para lo cual, se ha empleado la técnica del big $M$, explicada por Winston y Goldberg [12, frente a otras aproximaciones como el convex hull, que no tiene una fácil implementación debido a la posiblidad de mezclar distintos tipos de carro en el mismo equipo, lo que evita las relaciones uno a uno, necesarias en estos casos, que reducirían los tiempos de cómputo con estos métodos 2]. Sin embargo, se emplean distintos tamaños de parámetro big $M$ para acelerar la resolución.

El sistema se modela como un problema mixtoentero lineal, buscando una resolución lo más rápida posible para poder emplear el sistema en línea. Se plantea un modelo usando slots continuos, permitiendo una mayor flexibilidad en los tiempos de arranque frente a la discretización, más habitual en la actualidad. Además, en lugar de emplear las técnicas de preferencia más comunes, se mantiene la estrategia adoptada en los últimos trabajos del grupo 8], que hemos pasado a denominar preferencia predefinida (PP), donde los procesos están previamente ordenados aunque aún no tienen asignado ni un equipo ni un trabajo, por lo que quedan totalmente libres para ser asignados por el optimizador. Esto es, en la ejecución real de los procesos, unos acabarán ejecutándose antes que otros; este orden natural de los acontecimientos se incluye en el modelado del problema añadiendo un orden en el conjunto de slots, y obligando a que el instante de arranque de los mismos siga dicho orden. El desconocimiento del sistema se encuentra en la disposición que tendrán dichos slots en los equipos, y el tipo de producto que tratarán, que por lo tanto son las variables que quedan a elección del optimizador. Esta nueva técnica de preferencia añade la posibilidad de conocer cómo se relacionan los procesos entre sí, permitiendo reducir el número de restricciones que habría que incluir para identificar cómo afectan unos procesos a otros con las otras metodologías.

Frente a los scheduling que tienen un gran horizonte de predicción, en este trabajo se presenta un enfoque de horizonte deslizante, en el cual no se obliga a la esterilización de todos los carros, puesto que algunos de ellos podrán ser seleccionados en las siguientes iteraciones de la ejecución del problema. Para ello se fija un horizonte desde el instante actual, llamémosle robusto, para el cual se asegura la inclusión en algún proceso de esterilizado programado, de los carros que tienen su llegada prevista previa a dicho horizonte. El resto de carros podrán ser incluidos en los slots, si así lo estima oportuno el optimizador.

Inicialmente se determinan una serie de conjuntos:

- $\mathcal{H}$, es el conjunto de los tipos de latas, o referencias

- $\mathcal{I}$, son los carros llenos de producto que han sido liberados, o se prevé que lo sean dentro del periodo temporal que se analiza

- $\mathcal{J}$, el conjunto de grupos de carros que se introduce en el mismo esterilizador a la vez, también se puede denominar slot, o proceso de esterilizado, puesto que el grupo se ha unido para ser esterilizado

- $\mathcal{K}$, el conjunto de los autoclaves o esterilizadores

- $\mathcal{L}$, representa las líneas de sellado previas a la esterilización, son las líneas que liberan los carros llenos

Con estos conjuntos definidos, se pueden conseguir como parámetros de entrada al modelo los siguientes:

- $P_{i \in \mathcal{I}, h \in \mathcal{H}}$, es un parámetro binario que relaciona los carros con las referencias, cuando vale uno indica que el carro $i$ está lleno del tipo de producto $h$

- $Q_{i \in \mathcal{I}, l \in \mathcal{L}}$, relaciona los carros con las líneas de sellado, es un parámetro binario igual que el anterior. Cuando vale uno indica que el carro $i$ ha sido liberado por la línea de sellado $l$ 
- $o_{i \in \mathcal{I}}$, es un valor real que representa el instante temporal de liberación del carro $i$, almacena el tiempo en formato UNIX time ${ }^{1}$

- $r_{h \in \mathcal{H}}$, es un valor real que indica el tiempo mínimo fijado de duración de la fase de mantenimiento para la referencia $h$

- $\psi$, es un parámetro real que marca la duración mínima de la fase de calentamiento para cualquier proceso de esterilizado

- $\varphi$, es el tiempo máximo que se alarga una fase de calentamiento debido a la interacción con otro proceso de esterilizado

- $\xi$, representa el tiempo de enfriado, toma un valor real

- $\zeta$, es el número máximo de referencias permitidas en el mismo proceso de esterilizado o slot

- $\Gamma$, es la capacidad máxima de cada autoclave, es decir, el máximo número de carros

- $\gamma$, es el mínimo número de carros que se debe meter en cada proceso de esterilizado, que si bien puede ser uno, esto conlleva un gasto innecesario de recursos

- $\theta$, indica el tiempo máximo de espera de un carro desde que se sella hasta que se inicia el proceso de esterilizado

- $\delta$, es la máxima diferencia de tiempos de esterilizado entre los carros que están dentro de un mismo slot

- $\eta$, horizonte robusto, o límite temporal fijado que asegura que los carros que llegan antes son asignados a grupos

El problema de optimización que se desarrolla tiene cinco variables binarias que relacionan los conjuntos entre sí, o determinan comportamientos, y dos variables reales principales; aunque más adelante se definirán y explicarán una serie de variables auxiliares a fin de facilitar la codificación del problema. Inicialmente las variables binarias son:

- $X_{i \in \mathcal{I}, j \in \mathcal{J}}$, variable que indica que un carro $i$ está incluido en un grupo de carros o slot $j$

- $Y_{j \in \mathcal{J}, k \in \mathcal{K}}$, variable que señala en qué autoclave se introduce cada grupo de carros

- $U_{j \in \mathcal{J}}$, variable que indica si un proceso de esterilizado o slot se ejecuta o queda desierto

\footnotetext{
${ }^{1} \mathrm{El}$ formato UNIX time representa el tiempo como el número de segundos que han pasado desde el inicio del día 1 de enero de 1970 UTC
}

- $V_{j \in \mathcal{J}, h \in \mathcal{H}}$, variable que relaciona las referencias que han sido incluidas en un grupo de carros

- $W_{j \in \mathcal{J}, j^{\prime} \in \mathcal{J}}$, variable que indica que dos slots coinciden en el tiempo de calentamiento

Y las variables reales positivas son:

- $s_{j \in \mathcal{J}}$, instante de tiempo de inicio del proceso de esterilizado $j$, igual que el parámetro de tiempo de salida de los carros, el valor temporal se almacena con formato UNIX time

- $d_{j \in \mathcal{J}}$, duración de la fase de mantenimiento del proceso de esterilizado $j$

A continuación, se desarrollan las restricciones que definen el modelo. Primero se definen las restricciones de asignación. En la ecuación 1 se determina que un carro puede estar asignado como mucho a un grupo de carros, pero no tiene por qué estarlo, puesto que puede ser liberado de las líneas de sellado más tarde que $\eta$. Las ecuaciones 2 y 3 marcan los tamaños máximo y mínimo de cada proceso de esterilizado o slot. Después, en la ecuación 4 se indica que cada slot puede ser introducido como mucho en un autoclave, y en la ecuación 5 se muestra la relación entre la asignación de un grupo de carros a un slot, y la determinación si el slot se ejecuta. Por último, en la ecuación 6 se marca el máximo número distinto de referencias por cada grupo de carros.

$$
\begin{aligned}
& \sum_{j \in \mathcal{J}} X_{i, j} \leq 1 \quad \forall i \in \mathcal{I} \\
& \sum_{i \in \mathcal{I}} X_{i, j} \leq U_{j} \cdot \Gamma \quad \forall j \in \mathcal{J} \\
& \sum_{i \in \mathcal{I}} X_{i, j} \geq U_{j} \cdot \gamma \quad \forall j \in \mathcal{J} \\
& \sum_{k \in \mathcal{K}} Y_{j, k} \leq 1 \quad \forall j \in \mathcal{J} \\
& \sum_{k \in \mathcal{K}} Y_{j, k} \geq U_{j} \quad \forall j \in \mathcal{J} \\
& \sum_{h \in \mathcal{H}} V_{j, h} \leq \zeta \quad \forall j \in \mathcal{J}
\end{aligned}
$$

La variable que relaciona los tipos de latas con los grupos de carros se calcula mediante los carros incluidos en cada grupo, junto con el parámetro del tipo de referencia de cada carro $P_{i, h}$, como se 
muestra en las siguientes ecuaciones:

$$
\begin{gathered}
V_{j, h} \geq P_{i, h} \cdot X_{i, j} \\
\forall i \in \mathcal{I}, \forall j \in \mathcal{J}, \forall h \in \mathcal{H} \\
V_{j, h} \leq \sum_{i \in \mathcal{I}}\left(P_{i, h} \cdot X_{i, j}\right) \\
\forall j \in \mathcal{J}, \forall h \in \mathcal{H}
\end{gathered}
$$

Se define una variable auxiliar binaria $Z_{i \in \mathcal{I}, k \in \mathcal{K}}$ que relaciona los carros con los autoclaves en los que se introducen como parte de un grupo. Es decir, equivale a la conjunción entre las variables $X_{i, j}$ e $Y_{j, k}$; i.e. $Z_{i \in \mathcal{I}, k \in \mathcal{K}} \equiv X_{i, j \in \mathcal{J}} \wedge Y_{j, k}$. Esta variable sirve para restringir las asociaciones de carros que han llegado de ciertas líneas de sellado de ser introducidos en los autoclaves más lejanos, como puede verse en la ecuación 12 , donde sólo se permite que los carros que han sido liberados de las líneas del subconjunto $\mathcal{L}_{k} \subseteq \mathcal{L}$ se introduzcan en el autoclave $k$, entendiendo que el subconjunto $\mathcal{L}_{k}$ ha sido previamente definido como las líneas de sellado que pueden llevar carros al esterilizador $k$.

$$
\begin{aligned}
& \sum_{k \in \mathcal{K}} Z_{i, k} \leq \sum_{j \in \mathcal{J}} X_{i, j} \quad \forall i \in \mathcal{I} \\
& \sum_{i \in \mathcal{I}} Z_{i, k} \leq \Gamma \cdot \sum_{j \in \mathcal{J}} Y_{j, k} \quad \forall k \in \mathcal{K} \\
& Z_{i, k} \geq X_{i, j}+Y_{j, k}-1 \\
& \quad \forall i \in \mathcal{I}, \forall j \in \mathcal{J}, \forall k \in \mathcal{K} \\
& Z_{i, k} \leq \sum_{l \in \mathcal{L}_{k}} Q_{i, l} \quad \forall i \in \mathcal{I}, \forall k \in \mathcal{K}
\end{aligned}
$$

En las ecuaciones 13 y 14 se determina el tiempo de arranque de cada grupo de carros como superior al tiempo de liberación de cada carro incluido en el slot, e inferior al tiempo máximo de espera de todos ellos. Además, en la ecuación 15 se especifica que si un carro ha llegado antes del horizonte robusto $\eta$, debe ser incluido en algún slot.

$$
\begin{gathered}
s_{j} \geq o_{i}-M_{1} \cdot\left(1-X_{i, j}\right) \\
\forall i \in \mathcal{I}, \forall j \in \mathcal{J} \\
s_{j} \leq o_{i}+\theta+M_{1} \cdot\left(1-X_{i, j}\right) \\
\forall i \in \mathcal{I}, \forall j \in \mathcal{J} \\
\eta \leq o_{i}+M_{1} \cdot \sum_{j \in \mathcal{J}} X_{i, j} \quad \forall i \in \mathcal{I}
\end{gathered}
$$

Del mismo modo, en las ecuaciones 16 y 17 se fijan las restricciones a la duración mínima de los slots relativa a las referencias incluidas, y el tiempo máximo de diferencia entre las referencias introducidas que debe ser igual o menor que $\delta$.

$$
\begin{gathered}
d_{j} \geq r_{h}-M_{2} \cdot\left(1-V_{j, h}\right) \\
\forall j \in \mathcal{J}, \forall h \in \mathcal{H} \\
d_{j} \leq r_{h}+\delta+M_{2} \cdot\left(1-V_{j, h}\right) \\
\forall j \in \mathcal{J}, \forall h \in \mathcal{H}
\end{gathered}
$$

Con las ecuaciones anteriores, se han creado los grupos de carros, y se han asignado a los autoclaves. En las ecuaciones siguientes se ordenan dentro de los equipos, y se calculan las interferencias que se crean entre ellos. Para modelar la interferencia entre distintos procesos de esterilizado, se ha aproximado mediante una línea recta que parte del valor unitario si ambos procesos empiezan a la vez, y llega a valer cero si el ciclo de calentamiento del primero prácticamente ha finalizado cuando empieza el segundo, ver figura 2. Una vez que se tiene este ratio de superposición, se multiplica por el valor máximo que tendría la alteración de los ciclos de calentamiento, ver las ecuaciones de 20 a 22 más adelante.

Primero hay que indicar que el conjunto de grupos de carros, $\mathcal{J}$, se define como un conjunto ordenado, de manera que se puede decir que un miembro del conjunto precede o sucede a otro; y además, esta propiedad debe ser transitiva, de modo que si dos conjuntos se suceden, un tercero puede preceder a ambos, suceder al primero y preceder al segundo, o bien suceder a los dos, pero nunca suceder al segundo y preceder al primero al mismo tiempo. Además, se define una nueva variable auxiliar, $c_{j \in \mathcal{J}}$ que modela el tiempo de calentamiento de cada slot, y a su vez $e_{j \in \mathcal{J}, j^{\prime} \in \mathcal{J}}$ que sirve para calcular la interferencia que se provocan entre sí dos arranques concurrentes.

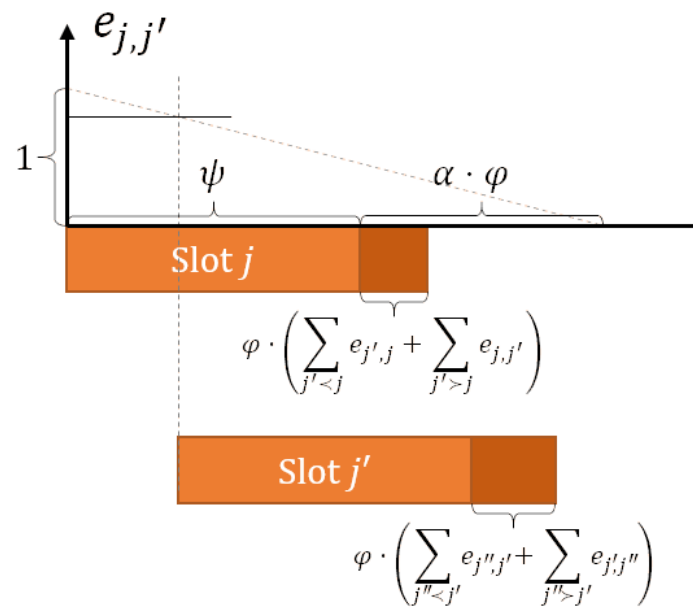

Figura 2: Representación gráfica de las interferencias entre las fases de calentamiento de los slots

En la ecuacion 18 se reproduce el orden dentro del 
conjunto al orden en el arranque de los slots. En la ecuacion 19 se indica el orden si dos slots están asignados al mismo autoclave, que determina que un slot no puede arrancar hasta que el anterior haya terminado, esto es, hasta que se ha calentado, se ha mantenido el tiempo necesario a la temperatura de consigna y se ha enfriado. En la ecuacion 20 se calcula el tiempo de calentamiento de un slot como el tiempo mínimo más el tiempo relativo a la interacción con los slots sucesivos y previos. En las ecuaciones 21 y 22 se aproxima el tiempo que se ve alterada cada fase de calentamiento debido a cada slot sucesivo, donde la constante $\alpha$, es un parámetro que sirve para ser más o menos restrictivo con la interacción entre dos slots, ver la figura 2 para más detalles.

$$
\begin{aligned}
& s_{j} \leq s_{j^{\prime}} \quad \forall j, j^{\prime} \in \mathcal{J}: j \prec j^{\prime} \\
& s_{j^{\prime}} \geq s_{j}+c_{j}+d_{j}+\xi \cdot U_{j} \\
&-M_{1} \cdot\left(2-Y_{j, k}-Y_{j^{\prime}, k}\right) \\
& \forall j, j^{\prime} \in \mathcal{J}, \forall k \in \mathcal{K}: j \prec j^{\prime} \\
& c_{j}=\psi \cdot U_{j}+\varphi \cdot\left(\sum_{j^{\prime} \in \mathcal{J}: j^{\prime} \prec j} e_{j^{\prime}, j}\right. \\
&\left.+\sum_{j^{\prime} \in \mathcal{J}: j^{\prime} \succ j} e_{j, j^{\prime}}\right) \\
& \forall j \in \mathcal{J} \\
& e_{j, j^{\prime}} \geq 1-\frac{s_{j^{\prime}}-s_{j}}{(\psi+\alpha \cdot \varphi)}-M_{3} \cdot\left(1-W_{j, j^{\prime}}\right) \\
& \forall j, j^{\prime} \in \mathcal{J}: j \prec j^{\prime} \\
& e_{j, j^{\prime}} \leq W_{j, j^{\prime}} \forall j, j^{\prime} \in \mathcal{J}: j \prec j^{\prime}
\end{aligned}
$$

En las ecuaciones 23 y 24 se restringe el inicio de un slot a producirse durante el tiempo de calentamiento si la variable binaria $W_{j, j}$ es igual a uno, o bien una vez que ha terminado si esta variable vale cero.

$$
\begin{gathered}
s_{j}+c_{j} \geq s_{j^{\prime}}-M_{1} \cdot\left(1-W_{j, j^{\prime}}\right) \\
\forall j, j^{\prime} \in \mathcal{J}: j \prec j^{\prime} \\
s_{j}+c_{j} \leq s_{j^{\prime}}+M_{1} \cdot W_{j, j^{\prime}} \\
\forall j, j^{\prime} \in \mathcal{J}: j \prec j^{\prime}
\end{gathered}
$$

En las ecuaciones de la 25 a la 28 se añaden restricciones en cuanto al orden de las posibles interacciones. Por ejemplo, en la ecuación 25 si un slot coincide en el calentamiento con otro, también debe coincidir con todos los intermedios.

$$
\begin{aligned}
& W_{j, j^{\prime}} \geq W_{j, j^{\prime \prime}} \\
& \forall j, j^{\prime}, j^{\prime \prime} \in \mathcal{J}: j \prec j^{\prime} \prec j^{\prime \prime} \\
& W_{j, j^{\prime \prime}} \leq W_{j^{\prime}, j^{\prime \prime}} \\
& \forall j, j^{\prime}, j^{\prime \prime} \in \mathcal{J}: j \prec j^{\prime} \prec j^{\prime \prime} \\
& W_{j, j^{\prime}} \leq U_{j} \\
& \forall j, j^{\prime} \in \mathcal{J}: j \prec j^{\prime} \\
& W_{j, j^{\prime}} \leq U_{j^{\prime}} \\
& \forall j, j^{\prime} \in \mathcal{J}: j \prec j^{\prime}
\end{aligned}
$$

Por último, se añade una restricción para poder incluir la posibilidad de que los autoclaves estén funcionando cuando se ejecute el problema de optimización. Para ello se emplea como parámetro el tiempo de finalización de los procesos de esterilización activos, $\tau_{k}$.

$$
s_{j} \geq \tau_{k} \cdot Y_{j, k} \quad \forall j \in \mathcal{J}, \forall k \in \mathcal{K}
$$

En los problemas de programación de tareas una función objetivo clásica es el tiempo final del proceso, o makespan (véase la ecuación 30); en este caso, sin embargo, el tiempo final del proceso no es importante, siempre y cuando se cumplan las restricciones de máxima espera, esto es las ecuaciones 14 y 15 , que aseguran un correcto procesamiento de las materias dentro de los plazos requeridos. Por otro lado, también es común la utilización de una función económica, que reduzca las pérdidas causadas por las paradas de las líneas de producción 6] o los costes de algún recurso, en este caso la función de coste requiere del modelado más concreto del comportamiento de las latas, lo cual quedaba fuera de este trabajo. Se ha obtado, finalmente, por maximizar la producción; i.e. máximizar el número de latas o carros que se procesan, véase la ecuación 31, asegurando un tiempo máximo final, $\beta$, fijado por el controlador de la sección de autoclavado, ecuación 32.

$$
\begin{gathered}
\text { makespan } \geq s_{j}+c_{j}+d_{j}+\xi \\
\forall j \in \mathcal{J} \\
\text { maximizar } \sum_{i \in \mathcal{I}, j \in \mathcal{J}} X i, j \\
\text { makespan } \leq \beta
\end{gathered}
$$

\section{RESULTADOS}

El problema ha sido codificado usando el lenguaje de programación de alto nivel Julia, y el paquete JuMP que permite emplear un lenguaje 
de programación basado en OPL [11] de manera similar a como lo hacen otros programas de optimización 44. El problema se resuelve usando CPLEX [7]. Para realizar las pruebas de manera adecuada se ha trabajado tanto con datos reales de planta como con distribuciones aleatorias de llegada de los carros. Se muestra un ejemplo de optimización con: la asignación de 150 carros; ordenados en 13 grupos de carros; con cinco líneas de sellado con sendas referencias; y, en diez autoclaves con una capacidad máxima de siete carros. Esto se traduce en un problema con más de 3800 variables binarias, más de 100 continuas, y casi 45000 restricciones.

Empleando como función de coste maximizar la producción, el problema se ejecuta en pocos minutos. Se pueden ver los resultados de las interacciones entre los slots en la tabla 1. En ella se muestra tanto el valor que toma la variable real $e_{j, j^{\prime}}$, como el valor total real debido a las interacción para cada slot dando a $\varphi$ el valor de 5 . Como puede verse para algunos slots sí se consigue mejorar la aproximación burda de estimar la máxima interferencia, sin embargo, puesto que la función de coste no incluye los tiempos de finalización, sino que se trata como una restricción más, algunos procesos de esterilizado coinciden totalmente en el arranque.

\section{DISCUSIÓN}

Se ha desarrollado un algoritmo que puede planificar las tareas en un horizonte temporal corto para poder adaptarse al entorno industrial cambiante. Tras varias pruebas, se ha comprobado que el sistema que calcula la interacción entre los procesos es útil cuando los tiempos de alteración son elevados, mientras que es más apropiado utilizar la aproximación máxima cuando los tiempos de variación no difieren mucho. Es decir, el aumento en la complejidad del problema, que conlleva un aumento en los tiempos de cálculo, no se ven compensados por soluciones mucho mejores si los tiempos de interacción son pequeños. Sin embargo, si estas interacciones influyen en gran medida en la solución final, no se debería obviar que el reparto de recursos depende del tiempo de coincidencia, y no sólo de la coincidencia en sí.

Gracias a la implementación en un lenguaje de programación de alto nivel en lugar de usando los programas de optimización, se ha mejorado mucho la interfaz con otros programas, de manera que se puede conectar el resultado directamente con la base de datos de la planta para poder recoger el estado actual de la misma, y devolver las recomendaciones para implementarlas o bien realizar una interfaz para mostrar al operario.
La metodología de precedencia predefinida se ha demostrado útil para tratar con interacciones de procesos, ya que se suprime una gran cantidad de restricciones frente a las metodologías clásicas.

\section{Agradecimientos}

Este trabajo ha sido financiado por el programa de innovación y desarrollo de la Unión Europea Horizonte 2020, proyecto CoPro (contrato $\left.\mathrm{n}^{\mathrm{o}} 723575\right)$ y por el Gobierno de España con fondos MINECO/FEDER bajo el proyecto InCo4In (PGC 2018-099312-B-C31). Del mismo modo, los autores desean extender su agradecimiento por el apoyo recibido desde el gobierno de Castilla y León y los fondos EU-FEDER (CLU 2017-09).

\section{English summary}

\section{OPTIMAL SCHEDULING OF BATCH PROCESSES WITH IN- TERDEPENDENT LENGTH}

\begin{abstract}
When similar pieces of equipment work concurrently sharing one resource, the working profiles can be affected by the consumption of the other equipment. Therefore, a scheduling problem arises in order to find the best temporal planification taking into account these alterations. This problem could get more complex if the profiles are not fixed, but depends on the products introduced in the equipment. In this work the authors present one tool to optimize the scheduling in a sterilization section inside a tuna canning plant, keeping in mind the alterations that the heating procedure of the autoclaves suffer if two or more autoclaves start at the same time. This tool has a moving horizon approach in order to be run iteratively, and to adapt the solution to the actual state of the plant.
\end{abstract}

Keywords: Autoclaves, RTO, rolling horizon, sterilization.

\section{Referencias}

[1] Andoni Amurrio, Ekain Azketa, J. Javier Gutiérrez, Mario Aldea y Jorge Parra. "Una revisión de técnicas para la optimización del despliegue y planificación de sistemas de 


\begin{tabular}{|c|c|c|c|c|c|c|c|c|c|c|c|c|c|c|}
\hline & $\mathrm{j} 1$ & $\mathrm{j} 2$ & j3 & $\mathrm{j} 4$ & $\mathrm{j} 5$ & $\mathrm{j} 6$ & j7 & $\mathrm{j} 8$ & j9 & $\mathrm{j} 10$ & $\mathrm{j} 11$ & $\mathrm{j} 12$ & $\mathrm{j} 13$ & total \\
\hline j1 & - & 1,0 & 0,0 & 0,0 & 0,0 & 0,0 & 0,0 & 0,0 & 0,0 & 0,0 & - & - & - & 5,00 \\
\hline $\mathrm{j} 2$ & - & - & 0,0 & 0,0 & 0,0 & 0,0 & 0,0 & 0,0 & 0,0 & 0,0 & 0,0 & - & - & 5,00 \\
\hline j3 & - & - & - & 0,0 & 0,0 & 0,0 & 0,0 & 0,0 & 0,0 & 0,0 & 0,0 & 0,0 & - & 0,00 \\
\hline $\mathrm{j} 4$ & - & - & - & - & 0,44 & 0,0 & 0,0 & 0,0 & 0,0 & 0,0 & 0,0 & 0,0 & 0,0 & 2,22 \\
\hline j5 & - & - & - & - & - & 0,95 & 0,0 & 0,0 & 0,0 & 0,0 & 0,0 & 0,0 & 0,0 & 6,98 \\
\hline j6 & - & - & - & - & - & - & 0,0 & 0,0 & 0,0 & 0,0 & 0,0 & 0,0 & 0,0 & 4,76 \\
\hline j7 & - & - & - & - & - & - & - & 1,0 & 1,0 & 1,0 & 1,0 & 1,0 & 0,0 & 25,00 \\
\hline j8 & - & - & - & - & - & - & - & - & 1,0 & 1,0 & 1,0 & 1,0 & 0,0 & 25,00 \\
\hline j9 & - & - & - & - & - & - & - & - & - & 1,0 & 1,0 & 1,0 & 0,0 & 25,00 \\
\hline j10 & - & - & - & - & - & - & - & - & - & - & 1,0 & 1,0 & 0,0 & 25,00 \\
\hline j11 & - & - & - & - & - & - & - & - & - & - & - & 1,0 & 0,0 & 25,00 \\
\hline j12 & - & - & - & - & - & - & - & - & - & - & - & - & 0,0 & 25,00 \\
\hline j13 & - & - & - & - & - & - & - & - & - & - & - & - & - & 0,00 \\
\hline
\end{tabular}

Tabla 1: Valores de interacción entre los slots, $j \in \mathcal{J}$

tiempo real distribuidos". En: Revista Iberoamericana de Automática e Informática industrial 16.3 (2019), págs. 249-263. DOI: 10 . 4995/riai.2019.10997

[2] Egon Balas. Disjunctive Programming. en. Cham: Springer International Publishing, 2018. DOI: 10.1007/978-3-030-00148-3.

[3] Pedro M. Castro, Iiro Harjunkoski e Ignacio E. Grossmann. "Discrete and continuoustime formulations for dealing with break periods: Preemptive and non-preemptive scheduling". En: European Journal of Operational Research 278.2 (2019). Advances in Data Envelopment Analysis, págs. 563-577. DOI: https://doi.org/10.1016/j.ejor. 2019. 04.025

[4] Iain Dunning, Joey Huchette y Miles Lubin. "JuMP: A Modeling Language for Mathematical Optimization". En: SIAM Review 59.2 (2017), págs. 295-320. DOI: $10.1137 /$ 15M1020575.

[5] Iiro Harjunkoski, Christos T. Maravelias, Peter Bongers, Pedro M. Castro, Sebastian Engell, Ignacio E. Grossmann, John Hooker, Carlos Méndez, Guido Sand y John Wassick. "Scope for industrial applications of production scheduling models and solution methods". En: Computers $\&$ Chemical Engineering 62 (2014), págs. 161-193. DOI: https: // doi . org / 10 . 1016/j . compchemeng . 2013.12.001.

[6] M. Kalliski, J. L. Pitarch, C. Jasch y C. de Prada. "Apoyo a la Toma de Decisión en una Red de Evaporadores Industriales". En: Revista Iberoamericana de Automática e Informática industrial 16.1 (2019), págs. 26-35. DOI: 10 . 4995 / riai . 2018 . 9233.
[7] CPLEX User's Manual. "IBM ILOG CPLEX Optimization Studio". En: Version 12 (2018), págs. 1987-2018.

[8] Carlos Gómez Palacín, Carlos Alberto Mendez y Cesar De Prada. "Slots Startup Synchronization with Shared Resources Dependency". En: Chemical Engineering Transactions 74 (2019), págs. 1321-1326.

[9] Cesar de Prada, Rogelio Mazaeda y Smaranda Podar. "Optimal Operation of a Combined Continuous-Batch Process". En: Computer Aided Chemical Engineering. Vol. 44. Elsevier, 2018, págs. 673-678.

[10] G Schilling y CC Pantelides. "A simple continuous-time process scheduling formulation and a novel solution algorithm". En: Computers \& Chemical Engineering 20 (1996), S1221-S1226.

[11] P. Van Hentenryck, L. Michel, L. Perron y J. -C. Régin. "Constraint Programming in OPL". En: Principles and Practice of Declarative Programming. Ed. por Gopalan Nadathur. Berlin, Heidelberg: Springer Berlin Heidelberg, 1999, págs. 98-116.

[12] Wayne L Winston y Jeffrey B Goldberg. Operations research: applications and algorithms. Vol. 3. Thomson/Brooks/Cole Belmont`eCalif Calif, 2004.

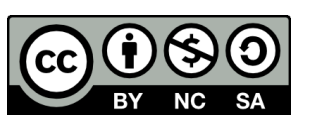
(c) 2019 by the authors. Submitted for possible open access publication under the terms and conditions of the Creative Commons Attribution CC BY-NC-SA 4.0 license (https://creativecommons.org/licenses/by-ncsa/4.0/deed.es). 